\title{
Spontaneous Currents in Josephson Devices
}

\author{
Z. Radović, ${ }^{1}$ L. Dobrosavljević-Grujić, ${ }^{2}$ B. Vujičić ${ }^{3}$ \\ ${ }^{1}$ Faculty of Physics, University of Belgrade, P.O. Box 368, 11001 Belgrade, Yugoslavia \\ ${ }^{2}$ Institute of Physics, P.O. Box 57, 11001 Belgrade, Yugoslavia \\ ${ }^{3}$ Faculty of Science, University of Montenegro, P.O. Box 211, 81000 Podgorica, Yugoslavia \\ To be published in Phys. Rev. B 60 (1 september 1999-I)
}

\begin{abstract}
The unconventional Josephson coupling in a ferromagnetic weak link between $d$-wave superconductors is studied theoretically. For strong ferromagnetic barrier influence, the unconventional coupling, with ground state phase difference across the link $0<\phi_{\mathrm{gs}} \leq \pi$, is obtained at small crystal misorientation of the superconducting electrodes, in contrast to the case of normal metal barrier, where it appears at large misorientations. In both cases, with decreasing temperature there is an increasing range of misorientations, where $\phi_{\mathrm{gs}}$ varies continuously between 0 and $\pi$. When the weak link is a part of a superconducting ring, this is accompanied by the flow of spontaneous supercurrent, of intensity which depends (for a given misorientation) on the reduced inductance $l=2 \pi L I_{c}(T) / \Phi_{0}$, and is non-zero only for $l$ greater than a critical value. For $l \gg 1$, another consequence of the unconventional coupling is the anomalous quantization of the magnetic flux.
\end{abstract}

\section{INTRODUCTION}

Magnetic scattering effects and $d$-wave pairing in superconductors may have similar manifestations, such as $\pi$-phase states and spontaneous currents in Josephson devices.1

The phase of the superconducting order parameter as a function of the momentum-space direction is different for $d$-wave and $s$-wave pairing states, since the latter has a single phase, whereas the $d$-wave state exhibits jumps of $\pi$ at the (110) lines. 6 The Josephson current through a junction depends on the phase difference $\phi$ between the superconductors on the either side, $I \sim \sin \phi$, and the negative sign to the prefactor of $I$ can be regarded as an additional phase shift of $\pi$. Superconducting rings containing an odd number of such $\pi$ shifts have the spontaneous magnetization $\pm \Phi_{0} / 2$, and quantized flux of $(n+1 / 2) \Phi_{0}$, where $n$ is an integer, providing that the product of the critical current and the self-inductance of the ring is $I_{c} L \gg \Phi_{0} / 2 \pi$.

Beside $d$-wave pairing, two alternative mechanisms for $\pi$-phase shifts at a conventional iunction have been proposed. One is the spin-flip scattering in the junction barrier containing paramagnetic impurities, 1 and the other is the indirect tunneling through a localized state, in which correlation effects produce a negative Josephson coupling between two superconducting grains.

A problem related to the first mechanism is the possibility of $\pi$-coupling in superconductor/ferromagnetic metal $(\mathrm{S} / \mathrm{F})$ weak links and multilayers, due to the presence of the exchange field in $\mathrm{F}$. 6 The characteristic oscillations of the critical temperature $T_{c}$ of multilayers as a function of the ferromagnetic layer thickness $d_{\mathrm{F}}$, predicted theoretically by Radović et al. were observed in $\mathrm{Nb} / \mathrm{Gd}$ multilayers by Strunk et al. 10 and by Jiang et al.11 and in $\mathrm{Nb} / \mathrm{CuMn}$ (superconductor/spinglass multilayers) by Mercaldo et al.12 While the oscillatory $T_{c}$ behavior was interpreted in terms of $\pi$-coupling in Refs. 11 and 12, in Ref. 10 it was attributed to the change from paramagnetic to ferromagnetic state with increasing $d_{\mathrm{F}}$. Recently, double minimum $T_{c}$ oscillations were observed on $\mathrm{Nb} / \mathrm{Co}$ and $\mathrm{V} / \mathrm{Co}$ multilayers by Obi et al.,13 and the second minimum was found to be consistent with the appearance of the $\pi$-phase.

In connection with the second mechanism, it was predicted that the anomalous flux quantization may appear with $50 \%$ probability in rings made from either disordered superconductors, or granular superconductors doped with paramagnetic impurities. The latter possibility was tested recently on uniform Mo rings doped with Fe, but the absence of the effect of paramagnetic impurities on flux quantization was found 14 This may be due to the fact that the phase shifts induced by the impurities do not survive averaging over different electron paths around the ring. It was suggested that a loop with nanometer size point contact, doped with magnetic impurities, might satisfy the requirement that electrons circling the loop are subject to the same exchange potential.

The Josephson effect in weak links with ferromagnetic metal barrier can be the direct probe of unconventional coupling. Tunneling properties of $s$-wave junctions with ferromagnetic insulator barrier have been studied thepretically by Kuplevakhskii and Fal'ko, 15 De Weert and Arnold,16 and more recently, by Tanaka and Kashiwaya.17 In the latter work the possibility of $\pi-$ shift was obtained. Tanaka and Kashiwaya studied separately the Josephson effect in anisotropic superconductor junctions with any symmetries and with a nonmagnetic insulating barrier.t8 This reference provides a general result from which several existing theories can be derived, and contains an extensive list 
of releyant references. Short weak links and $d$-wave junctions with normal metal barrier were studied by Barash et al.19.20 These authors find an essentially nonharmonic current-phase relation $I(\phi)$ at low temperature, and a flow of spontaneous supercurrents in superconducting ring interrupted by $\pi-$ junctions. Recently, Fogelstrom et al. have considered pinhole junctions in $d$-wave superconductors and have shown that at low temperature the ground state phase difference at the junction may vary continuously between 0 and $\pi 21$

In the present work, we show how new possibilities for unconventional coupling in S/F/S Josephson devices result from the combined effects of exchange field and $d$-wave pairing. We consider a Josephson weak link in the clean limit, with thin and short ferromagnetic (or normal) metal barrier. The interfaces between the barrier and the superconducting electrodes are assumed fully transparent. Two-dimensional (2D) superconductivity and $d_{x^{2}-y^{2}}$ symmetry of the order parameter are considered.22 Assuming the barrier perpendicular to the $a-b$ planes of superconducting electrodes, the influence of their relative orientation is studied. For comparison some results for $3 D s$-wave case are included. In Section II, we give a brief overview of the quasiclassical theory of superconductivity in presence of exchange field and for anisotropic pairing interaction, which we apply in the following. In Section III, we calculate the quasiclassical Green's functions for the weak link to obtain the supercurrent as a function of the phase difference at the contact. When the weak link is a part of a superconducting ring, the ground state phase difference is calculated and the conditions for the flow of spontaneous supercurrent and anomalous flux quantization are obtained. Section IV contains a discussion of the results and a brief conclusion.

\section{QUASICLASSICAL EQUATIONS}

A microscopic approach powerful enough to deal with superconductivity in restricted geometries is the Eilenberger quasiclassical theory of superconductivity 23 To calculate the critical current of $d$-wave $\mathrm{S} / \mathrm{F} / \mathrm{S}$ contact, we generalize the methor developed for the superconductor/normal metal/superconductor ( $\mathrm{S} / \mathrm{N} / \mathrm{S}$ ) contact by Syidzinskii and Likharev 2425 applied to S/F/S contact for $s$-wave pairing case by Buzdin et al. . $^{6}$ and by Demler et al. 9

The basic equations of the Eilenberger quasiclassical theory of superconductivity, in the presence of an exchange energy $h$, are

$$
\left[i \omega_{n} \hat{\tau}_{3}-\hat{\boldsymbol{\Delta}}+h \hat{\sigma}_{z} \hat{1}, \hat{g}\right]+i \mathbf{v}_{0} \cdot \nabla \hat{g}=0
$$

with normalization condition,

$$
\hat{g}^{2}=-\pi^{2} \hat{1}
$$

where $\omega_{n}=\pi T(2 n+1)$ are the Matsubara frequencies $\left(\hbar=k_{B}=1\right)$, $\mathbf{v}_{0}$ is the Fermi velocity, and $\hat{g}=\hat{g}\left(\mathbf{v}_{0}, \mathbf{R} ; \omega_{n}\right)$ is the quasiclassical Gorkov's Green's function integrated over energy. Explicitly,

$$
\hat{g}=\pi\left[\begin{array}{cccc}
-i g_{\uparrow} & 0 & 0 & f_{\uparrow \downarrow} \\
0 & -i g_{\downarrow} & -f_{\downarrow \uparrow} & 0 \\
0 & f_{\uparrow \downarrow}^{+} & i g_{\downarrow} & 0 \\
-f_{\downarrow \uparrow}^{+} & 0 & 0 & i g_{\uparrow}
\end{array}\right],
$$

the gap matrix is

$$
\hat{\boldsymbol{\Delta}}=\left[\begin{array}{cc}
0 & i \sigma_{y} \Delta\left(\mathbf{v}_{0}, \mathbf{R}\right) \\
i \sigma_{y} \Delta^{*}\left(-\mathbf{v}_{0}, \mathbf{R}\right) & 0
\end{array}\right],
$$

and the self-consistency equation is

$$
\Delta\left(\mathbf{v}_{0}, \mathbf{R}\right)=\pi N T \sum_{\omega_{n}}\left\langle V\left(\mathbf{v}_{0}, \mathbf{v}_{0}^{\prime}\right) \frac{f_{\downarrow \uparrow}\left(\mathbf{v}_{0}^{\prime}, \mathbf{R} ; \omega_{n}\right)+f_{\uparrow \downarrow}\left(\mathbf{v}_{0}^{\prime}, \mathbf{R} ; \omega_{n}\right)}{2}\right\rangle,
$$

where $V\left(\mathbf{v}_{0}, \mathbf{v}_{0}^{\prime}\right)$ is the pairing interaction. The supercurrent density is given by

$$
\mathbf{j}(\mathbf{R})=-2 i e \pi N T \sum_{\omega_{n}}\left\langle\mathbf{v}_{0} \frac{g_{\uparrow}+g_{\downarrow}}{2}\right\rangle
$$

where $\langle\cdots\rangle$ denotes the angular averaging over the Fermi surface, $N$ being the density of states at the Fermi surface. 
In the following, we use the notation $f_{\downarrow \uparrow}=f, f_{\uparrow \downarrow}^{+}=f^{+}, g_{\downarrow}=g$ for the set of Green's function for one spin direction (down with respect to the exchange field orientation). They satisfy the scalar equations

$$
\begin{gathered}
2\left(w_{n}+i h\right) f+\mathbf{v}_{\mathbf{0}} \nabla f=2 g \Delta, \\
2\left(w_{n}+i h\right) f^{+}-\mathbf{v}_{\mathbf{0}} \nabla f^{+}=2 g \Delta^{*}, \\
\mathbf{v}_{\mathbf{0}} \nabla g=\Delta^{*} f-f^{+} \Delta .
\end{gathered}
$$

For the opposite spin direction, the corresponding set of Green's functions is obtained by changing $h \rightarrow-h$.

\section{SOLUTIONS}

We solve the quasiclassical equations for a $d$-wave $\mathrm{S} / \mathrm{F} / \mathrm{S}$ junction, where $\mathrm{S}$ is an anisotropic superconductor with $d_{x^{2}-y^{2}}$ symmetry, and $\mathrm{F}$ is a monodomain ferromagnetic metal with constant exchange energy $h$.

We assume both $\mathrm{S}$ and $\mathrm{F}$ metals clean, with same dispersion relations and with same Fermi velocity $\mathbf{v}_{\mathbf{0}}$. Electron scattering on impurities in $\mathrm{S}$ can be neglected if $l \gg \xi_{0}$, and in $\mathrm{F}$ if $h \gg v_{0} / l$, where $l$ is the electron mean free path and $\xi_{0}$ the BCS superconducting coherence length.

The thin and short barrier, of thickness $d$, is assumed perpendicular to the $\hat{a}$ axis in the $\hat{a}-\hat{b}$ plane of the lhs monocrystal $\mathrm{S}_{\mathrm{L}}$, which may be misoriented with respect to the rhs one, $\mathrm{S}_{\mathrm{R}}$, their $\hat{a}$ axes making an angle $\theta$ (Fig. 1). For anisotropic pairing, the pair potential and the shape of quasiparticle spectra depend on the misorientation. For $d_{x^{2}-y^{2}}$ symmetry,22 the pairing interaction and the pair potential in $\mathrm{S}_{\mathrm{L}}$ are $V\left(\mathbf{v}_{\mathbf{0}}, \mathbf{v}_{\mathbf{0}}^{\prime}\right) \propto \cos 2 \varphi \cos 2 \varphi^{\prime}$ and $\Delta\left(\mathbf{v}_{\mathbf{0}}\right) \propto \cos \mathbf{2} \varphi$, respectively, where $\varphi$ is the angle the quasiparticle momentum makes with the $\hat{a}$ axis. Similarly, in $\mathrm{S}_{\mathrm{R}}, \Delta\left(\mathbf{v}_{\mathbf{0}}\right) \propto \cos 2(\varphi-\theta)$. Assuming $2 \mathrm{D}$ nature of HTS with $d$-wave pairing, we take cylindrical Fermi surface for both metals, whereas for 3D $s$-wave case spherical Fermi surface is taken.

To solve Eqs. (2.7)-(2.9), we take in $\mathrm{F}$, where $h=$ const., $\Delta=0$, but $f \neq 0$ due to proximity of $\mathrm{S}$. In $\mathrm{S}$, where $h=0$, we take the order parameter on two sides of the barrier in the form $\Delta_{\mathrm{L}, \mathrm{R}}=\Delta_{\mathrm{L}, \mathrm{R}}(\varphi) e^{ \pm i \phi / 2}$, where $\phi$ is the phase difference at the contact, $\Delta_{\mathrm{L}}(\varphi)=\Delta_{0} \cos 2 \varphi, \Delta_{\mathrm{R}}(\varphi)=\Delta_{0} \cos 2(\varphi-\theta)$ for $d$-wave $\left(\Delta(\varphi)=\Delta_{0}\right.$ for $s$-wave $)$ pairing 26 Assuming $d / \xi_{0}<1$, we neglect the spatial variation of $\Delta$, which in principle could be obtained from the self-consistency equation (2.5).

Far from the barrier, $f$ and $g$ approach their respective bulk values

$$
\left\langle f_{\mathrm{L}, \mathrm{R}}\right\rangle=\left\langle f_{\mathrm{L}, \mathrm{R}}^{+}\right\rangle^{*}=\Delta_{\mathrm{L}, \mathrm{R}} / \tilde{\omega}_{n \mathrm{~L}, \mathrm{R}},
$$

and

$$
\left\langle g_{\mathrm{L}, \mathrm{R}}\right\rangle=\omega_{n} / \tilde{\omega}_{n \mathrm{~L}, \mathrm{R}} .
$$

where

$$
\tilde{\omega}_{n \mathrm{~L}, \mathrm{R}}=\sqrt{\omega_{n}^{2}+|\Delta|_{\mathrm{L}, \mathrm{R}}^{2}}
$$

Choosing the $x$-direction along $\hat{a}_{\mathrm{L}}$, we look for the solution at the rhs, $x \geq d / 2$,

$$
f_{\mathrm{R}}=\left\langle f_{\mathrm{R}}\right\rangle+\bar{f}_{\mathrm{R}} e^{-\alpha_{\mathrm{R}} x}, \quad g_{\mathrm{R}}=\left\langle g_{\mathrm{R}}\right\rangle+\bar{g}_{\mathrm{R}} e^{-\alpha_{\mathrm{R}} x} .
$$

Similarly, at lhs, $x \leq d / 2$,

$$
f_{\mathrm{L}}=\left\langle f_{\mathrm{L}}\right\rangle+\bar{f}_{\mathrm{L}} e^{\alpha_{\mathrm{L}} x}, \quad g_{\mathrm{L}}=\left\langle g_{\mathrm{L}}\right\rangle+\bar{g}_{\mathrm{L}} e^{\alpha_{\mathrm{L}} x} .
$$

From Eqs. (2.7)-(2.9) we find

$$
\bar{f}_{\mathrm{R}}=\bar{g}_{\mathrm{R}} \frac{\Delta_{\mathrm{R}}}{\omega_{n}-\alpha_{\mathrm{R}} \mathrm{v}_{x} / 2}, \quad \bar{f}_{\mathrm{L}}=\bar{g}_{\mathrm{L}} \frac{\Delta_{\mathrm{L}}}{\omega_{n}+\alpha_{\mathrm{L}} \mathrm{v}_{x} / 2},
$$

with 


$$
\alpha_{\mathrm{L}, \mathrm{R}}=\frac{2 \tilde{\omega}_{n \mathrm{~L}, \mathrm{R}}}{\mathrm{v}_{x}}=\frac{2 \tilde{\omega}_{n \mathrm{~L}, \mathrm{R}}}{\mathrm{v}_{0} \cos \varphi}
$$

For $\mathrm{F}, \Delta=0$, and the solutions are

$$
\begin{array}{r}
f=C \exp \left\{\frac{-2\left(\omega_{n}+i h\right) x}{\mathrm{v}_{\mathrm{x}}}\right\}, \\
f^{+}=C^{+} \exp \left\{\frac{2\left(\omega_{n}+i h\right) x}{\mathrm{v}_{\mathrm{x}}}\right\},
\end{array}
$$

and $g=$ const. for $|x| \leq d / 2$. Note that $f^{+}=f\left(-\mathbf{v}_{0}, \Delta^{*}\right)$. Assuming a transparent boundary between two metals, we use the continuity conditions for $g$ and $f$ at the barrier interfaces, $x= \pm d / 2$. We find the normal Green's function in the barrier

$$
g=\frac{\omega_{n}}{\tilde{\omega}_{n \mathrm{~L}}}-\frac{\left(\omega_{n}+\tilde{\omega}_{n \mathrm{~L}}\right)\left[\left(\omega_{n}-\tilde{\omega}_{n \mathrm{R}}\right) \Delta_{\mathrm{L}}(\varphi)-\left(\omega_{n}-\tilde{\omega}_{n \mathrm{~L}}\right) \Delta_{\mathrm{R}}(\varphi) \exp (i \chi)\right]}{\tilde{\omega}_{n \mathrm{~L}}\left[\left(\omega_{n}-\tilde{\omega}_{n \mathrm{R}}\right) \Delta_{\mathrm{L}}(\varphi)-\left(\omega_{n}+\tilde{\omega}_{n \mathrm{~L}}\right) \Delta_{\mathrm{R}}(\varphi) \exp (i \chi)\right]},
$$

where

$$
\chi=\phi+\frac{Z}{\cos \varphi}-\frac{2 i \omega_{n} d}{\mathrm{v}_{0} \cos \varphi}
$$

and

$$
Z=\frac{2 h d}{\mathrm{v}_{0}}
$$

is the parameter measuring the ferromagnetic barrier influence. The temperature dependence of $\Delta_{0}=\Delta_{0}(T)$ is quite similar to the BCS one, and can be approximated by

$$
\Delta_{0}(T)=\Delta_{0}(0) \tanh \left(1.74 \sqrt{T_{c} / T-1}\right) .
$$

The only difference is that in the case of $d$-wave pairing $\Delta_{0}(0) / \Delta_{0}^{B C S}(0)>122$

From the above results it is easy to obtain the corresponding ones for $d$-wave junction with normal metal barrier, putting $h \rightarrow 0$, and for $s$-wave junction, taking isotropic pair potential $\Delta^{\mathrm{L}}=\Delta^{\mathrm{R}}=\Delta_{0}$, with ferromagnetic $(h \neq 0)$ or normal metal $(h=0)$ barrier.

\section{A. Supercurrents}

In $\mathrm{S} / \mathrm{N} / \mathrm{S}$ case, for $d$-wave pairing the ground state phase difference on the contact is zero, as for the $s$-wave pairing, if two $\mathrm{S}$ monocrystals have the same orientation with respect to the barrier. This may not be the case for $\mathrm{S} / \mathrm{F} / \mathrm{S}$ contacts, and we calculate the supercurrent as a function of the barrier exchange field intensity, as well as the function of the orientation.

Taking the current direction along $\hat{a}_{L}$, from Eq. (2.6) we get for $2 \mathrm{D} d$-wave case

$$
I=I_{0} t \sum_{\omega_{n}, \sigma} \int_{1}^{\infty} \frac{\Im\left[g_{\sigma}(u)+g_{\sigma}(-u)\right]}{u^{2} \sqrt{u^{2}-1}} \mathrm{~d} u,
$$

where $u=1 / \cos \varphi$ and $\sigma=\downarrow, \uparrow$. Green's function $g_{\downarrow}$ is given by Eq. (3.9), and $g_{\uparrow}(h)=g_{\downarrow}(-h)$. Eq. (3.13) gives the supercurrent through the barrier of the area $S, I=j S$ as a function of $\phi, \theta$, temperature $T$ via $t=T / \Delta_{0}(T)$, and of parameters measuring the influence of the $\mathrm{F}$ barrier $Z$ and $\tilde{d}=d / \xi_{0}(0)$, where $\xi_{0}(0)=\mathrm{v}_{0} / \pi \Delta_{0}(0)$. Note that $Z \sim \tilde{d} h / \Delta_{0}(0)$. The temperature dependent normalizing current is

$$
I_{0}=\frac{2 \Delta_{0}(T)}{e R_{\mathrm{N}}}
$$

where the normal resistance is given by $R_{\mathrm{N}}^{-1}=e^{2} \mathrm{v}_{0} N S, N$ being the density of states at the Fermi surface.

In the limit $T \rightarrow T_{c}$, numerical calculations show that Eq. (3.13) gives, as expected, the Josephson relation 


$$
I=I_{c} \sin \phi,
$$

where the sign and magnitude of $I_{\text {mepend }}$ den $T, \theta$ and $Z$.

For $3 \mathrm{D}$ case and $s$-wave pairing, $\mathrm{\theta}$ Eq. (3.9) reduces to

$$
g=\frac{\omega_{n} \cos \frac{\chi}{2}+i \tilde{\omega}_{n} \sin \frac{\chi}{2}}{\tilde{\omega}_{n} \cos \frac{\chi}{2}+i \omega_{n} \sin \frac{\chi}{2}},
$$

where

$$
\tilde{\omega}_{n}=\sqrt{\omega_{n}^{2}+\left|\Delta_{0}\right|^{2}},
$$

and Eq. (2.6) gives

$$
I=\frac{\pi}{4} I_{0} t \sum_{\omega_{n}, \sigma} \int_{1}^{\infty} \frac{\Im\left[g_{\sigma}(u)+g_{\sigma}(-u)\right]}{u^{3}} \mathrm{~d} u,
$$

where the same notation as in Eq. (3.13) is used.

In general the supercurrents in the Josephson junctions and weak links are carried by the Andreev bound states in the barrier. 1827 In the present case the spectrum of these states has been calculated from the analytical continuation of the above Green's functions $g_{\sigma} .88$

\section{B. Magnetic flux}

For a superconducting ring containing one Josephspn junction, the ground state phase difference $\phi_{\mathrm{gs}}$ at the junction can be obtained by minimizing the reduced energy 29

$$
\tilde{W}(\phi)=\frac{1}{I_{c}} \int_{0}^{\phi} I\left(\phi^{\prime}\right) \mathrm{d} \phi^{\prime}+\frac{1}{2} l^{-1}\left(\phi-\phi_{\mathrm{e}}\right)^{2},
$$

where $I(\phi)$ is calculated from Eq. (3.13), or Eq. (3.18), $\tilde{W}=W /\left(\Phi_{0} I_{c} / 2 \pi\right), \Phi_{0}$ is the flux quantum, and the reduced inductance is

$$
l=\frac{2 \pi}{\Phi_{0}} I_{c} L
$$

with $I_{c}=I_{c}(T, \theta, Z)$. For $l^{-1}=0$, the ground state condition reduces to $I\left(\phi_{\mathrm{gs}}\right)=0, I^{\prime}\left(\phi_{\mathrm{gs}}\right)>0$.

In the limit $T \rightarrow T_{c}$, where the current-phase relation is harmonic, Eq. (3.15), from Eq. (3.19) it follows

$$
\sin \phi_{\mathrm{gs}}+l^{-1}\left(\phi_{\mathrm{gs}}-\phi_{\mathrm{e}}\right)=0 .
$$

The phase $\phi_{\mathrm{e}}=2 \pi \Phi_{\mathrm{e}} / \Phi_{0}$ represents the reduced external magnetic flux through the ring. In the ground state, the total magnetic flux

$$
\Phi=\frac{\Phi_{0}}{2 \pi} \phi_{\mathrm{gs}}
$$

is related to the spontaneous supercurrent $I_{\mathrm{gs}}=I\left(\phi_{\mathrm{gs}}\right)$ by

$$
\Phi=\Phi_{\mathrm{e}}-\frac{\Phi_{0}}{2 \pi} l I_{\mathrm{gs}} .
$$

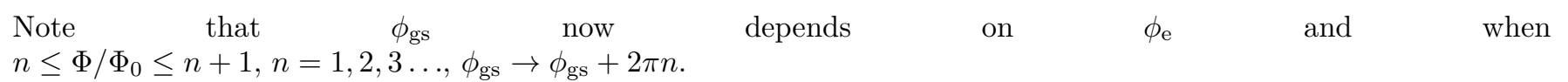




\section{RESULTS AND CONCLUSION}

To illustrate the consequences of unconventional coupling we present the results of numerical calculations for typical cases, low temperature, $t=0.05\left(T / T_{c} \approx 0.1\right)$ and high temperature, $t=5\left(T / T_{c} \approx 0.9\right)$, for strong influence of the ferromagnetic barrier $(Z=3)$ and for the normal metal barrier $(Z=0)$.

For $d$-wave pairing, $I(\phi)$ dependence is much more complex than in $s$-wave pairing case, even in the absence of exchange field. Besides deformations of sinusoidal curves at low $T$, the shape and sign of $I(\phi)$ depend very much on the misorientation angle $\theta$, both for normal and ferromagnetic metal barriers. The low $T$ deformations of $I(\phi)$ are reminiscence of discontinuities at $T=0$. At zerg temperature, for $Z=0$ the current turns out to be discontinuous at $\phi=\pi$ for $\theta=0$, as in the $s$-wave pairing case.25 For $\theta=\pi / 2$, the current jump is at $\phi=0$ (or $2 \pi$ ), and for $\theta=\pi / 4$ smaller jumps appear both at $\phi=0$ and $\pi$, similarly to the results of Ref. 19. With increasing temperature, $I(\phi)$ becomes less deformed tending to sinusoidal variations. Similar conclusions hold for $Z=3$. The dependence $I(\theta)$ persists in the presence of exchange field, as a strong evidence of $d$-wave pairing. This is shown in Fig. 2 for the characteristic values of $Z, t$ and $\theta$.

In the absence of external magnetic field, for $\theta=0$ the ground state phase difference $\phi_{\mathrm{gs}}$ at the $\mathrm{S} / \mathrm{F} / \mathrm{S}$ weak link is always zero or $\pi$, both for 3D $s$-wave and for $2 \mathrm{D} d$-wave pairing. For weak influence of the ferromagnetic barrier, $Z \lesssim 1, \phi_{\mathrm{gs}}=0$ and for stronger influence, $1 \lesssim Z \lesssim 4$, there is a $\pi$-shift, $\phi_{\mathrm{gs}}=\pi$ (Fig.3). Larger values of $Z$, for which $\phi_{\mathrm{gs}}=0$ again, would correspond to the decoupling of $\mathrm{S}$ electrodes. 30 For $\theta=\pi / 2, \phi_{\mathrm{gs}}=0 \rightarrow \phi_{\mathrm{gs}}=\pi$ and vice versa. These results are temperature independent. For $s$-wave tunnel junctions with ferromagnetic insulating barrier it is also found that $\phi_{\mathrm{gs}}$ changes from 0 to $\pi$ as the exchange interaction is enhanced. 27 In weak links, for 2D $d$-wave pairing we find that $\phi_{\mathrm{gs}}$ varies between 0 and $\pi$, depending on $\theta$ and $Z$. This variation is monotonous at low temperature, whereas at high temperature we find step function-like jumps at $\theta=\pi / 4$. In the vicinity of $\theta=\pi / 4$, depending on $Z$ and $\theta$ the transition from some intermediate value, $0<\phi_{\mathrm{gs}}<\pi$, to $\phi_{\mathrm{gs}}=0$ or to $\phi_{\mathrm{gs}}=\pi$ may occur with the change of temperature, similar to the 0 to $\pi$ transition found for $d$-wave tunnel junction, 20 see Figs. 4(a) and $4(\mathrm{c})$. For $d$-wave pinhole junctions a range of crystal orientations, where $\phi_{\mathrm{gs}}$ varies from 0 to $\pi$, is also found at low $T .21$

For superconducting rings with sufficiently large reduced inductance $l$, interrupted by the S/F/S link, the unconventional coupling is also found, with nonzero $\phi_{\mathrm{gs}}<\pi$, which rapidly decreases with increasing $l^{-1}$, Figs. 4(a) and $4(\mathrm{c})$. We note that at high temperature, $t>0.5, \phi_{\mathrm{gs}} \equiv 0$ for $l^{-1} \geq 1$.

The appearance of spontaneous current $I_{g s}(\theta)$ in superconducting rings for external magnetic flux $\Phi_{\mathrm{e}}=0$ is illustrated in Figs. 4(b) and 4(d) for several values of the reduced inductance. The flow of spontaneous supercurrent is the consequence of the misorientation effect, or of the exchange field influence, whenever $\phi_{g s} \neq 0$. This is a generalization of the spin-flip induced effect predicted by Bulaevskii et al. for conventional superconductor rings with junction barrier doped with paramagnetic impurities. 1 The highest values of spontaneous currents, corresponding to $\theta=\pi / 2$ for $Z=0$, and to $\theta=0$ for $Z=3$, strongly depend on the reduced inductance. Maximum values, equal to the critical currents, correspond to $l^{-1} \sim 1$ at low $T$, and to $l^{-1}<1$ at high $T$ (Fig. 5). It is important to point out that the spontaneous currents appear only for sufficiently large reduced inductance, greater than some critical value $l_{c}$, in contrast to the result of Barash et al. for $\mathrm{S} / \mathrm{N} / \mathrm{S}$ junctions at $T=0.19$ At high $T, t>0.5$, where the current-phase relation is harmonic, we obtain an universal curve below $l_{c}^{-1}=1$, as in the case of tunnel junctions.t At low $T$, the spontaneous currents flow is obtained in a wider range of $l^{-1}$, the shape of the curves and $l_{c}$ depending on the barrier influence $Z$ and on the type of pairing symmetry.

In the presence of external magnetic flux, $\Phi_{\mathrm{e}} \neq 0$, the flow of spontaneous current leads to the anomalous flux quantization in the ring, both for $\mathrm{S} / \mathrm{N} / \mathrm{S}$ and $\mathrm{S} / \mathrm{F} / \mathrm{S}$ junctions. In the first case, $Z=0$, this occurs in the vicinity of $\theta=\pi / 2$ and in the second case, $Z=3$, in the vicinity of $\theta=0$. The effect of half magnetic flux quantization, $\Phi / \Phi_{0}=1 / 2,3 / 2, \ldots$, pronounced for $l^{-1}$ small, becomes smeared out and eventually lost for larger $l^{-1}$ (Fig. 6).

Since $l^{-1} \propto 1 / I_{c}(T)$ rapidly increases with increasing temperature, the unconventional coupling effects, such as the spontaneous supercurrent flow and the anomalous flux quantization exist only below some temperature characteristic for the given device.

In conclusion, the spontaneous supercurrent flow in a HTS ring with ferromagnetic weak link provides new possibilities for experimental observation of the unconventional Josephson coupling, $0<\phi_{\mathrm{gs}} \leq \pi$. Due to the presence of the exchange field in the barrier, it may appear, manifested by the anomalous flux quantization, without the misorientation of the crystals in two superconducting electrodes. However, the exchange field effect do not mask the difference between $s$ and $d$-wave pairing, since the coupling depends on the misorientation. We emphasize that the unconventional coupling effects in the weak links are much more diverse than in the tunnel junctions, which display in the ground state only 0 or $\pi$ phase difference. 
${ }^{1}$ L. Dobrosavljević-Grujić and Z. Radović, in Spectroscopic Studies of Superconductors, Proc. SPIE v. 2696, 428 (1996).

${ }^{2}$ D. J. van Harlingen, Rev. Mod. Phys. 67, 515 (1995).

${ }^{3}$ C. C. Tsuei et al., Phys. Rev. Lett. 73, 593 (1994).

${ }^{4}$ L. N. Bulaevskii, V. V. Kuzii, and A. A. Sobyanin, Zh. Eksp. Teor. Fiz., Pis'ma 25, 314 (1977) [JETP Lett. 25, 290 (1977)].

${ }^{5}$ B. I. Spivak and S. Kivelson, Phys. Rev. B 43, 3740 (1991).

${ }^{6}$ A. I. Buzdin, L. N. Bulaevskii, and S. V. Paniukov, Zh. Eksp. Teor. Fiz., Pis'ma 35, 147 (1982).

${ }^{7}$ Z. Radović, M. Ledvij, L. Dobrosavljević-Grujić, A. I. Buzdin, and J. R. Clem, Phys. Rev. B 44, 759 (1991); Z. Radović and

L. Dobrosavljević-Grujić, in Superconducting Superlattices and Multilayers, Proc. SPIE v. 2157, 240 (1994).

${ }^{8}$ V. Prokić, A. I. Buzdin and L. Dobrosavljević-Grujić, Phys. Rev. B 59, 587 (1999).

${ }^{9}$ E. A. Demler, G. B. Arnold, and M. R. Beasley, Phys. Rev. B 55, 15174 (1998).

${ }^{10}$ C. Strunk, C. Sürgers, U. Pashen, and H. v. Löhneysen, Phys. Rev. B 49, 4053 (1994).

${ }^{11}$ J. S. Jiang, D. Davidović, Daniel H. Reich, and C. L. Chien, Phys. Rev. Lett. 74, 314 (1995).

${ }^{12}$ L. V. Mercaldo, C. Attanasio, C. Coccorese, L. Maritato, S. L. Prischepa, and M. Salvato, Phys. Rev. B 53, 14040 (1996).

${ }^{13}$ Y. Obi, M. Ikebe, T. Kubo, and H. Fujimori, Physica C, Proceedings of the First Euroconference on Anomalous Complex Superconductors, ACS'98, Crete-Greece, Sept. 26 - Oct. 3, 1998.

${ }^{14}$ D. Davidović and M. Tinkham, Phys. Rev. B 56, 5132 (1997).

15 S. V.Kuplevakhskĩ and I. I. Fal'ko, Fiz. Met. Metalloved. 7168 (1991) [Phys. Met. Metall. (USSR) 71, 65 (1991)].

${ }^{16}$ M. J. De Weert and G. B. Arnold, Phys. Rev. B 39, 11307 (1989).

17 Y. Tanaka and S. Kashiwaya, Physica C 274, 357 (1997).

${ }^{18}$ Y. Tanaka and S. Kashiwaya, Phys. Rev. B 56, 892 (1997).

${ }^{19}$ Yu. S. Barash, A. V. Galaktionov, and A. D. Zaikin, Phys. Rev B 52, 665 (1995).

${ }^{20}$ Yu. S. Barash, H. Burkhardt, and D. Rainer, Phys. Rev. Lett. 77, 4070 (1996).

${ }^{21}$ M. Fogelstrom, S. Yip, and J. Kurkijavari, Physica C 294, 289 (1998).

${ }^{22}$ H. Won and K. Maki, Phys. Rev. B 49, 1397 (1993).

${ }^{23}$ J. A. X. Alexander et al., Phys. Rev. B 31, 5811 (1985); G. Eilenberger, Z. Phys. 214, 195 (1968).

${ }^{24}$ A. V. Svidzinskii, Spatially Inhomogeneous Problems in the Theory of Superconductivity (Nauka, Moskva, 1982).

${ }^{25}$ K. K. Likharev, Rev. Mod. Phys. 51, 101 (1979).

${ }^{26}$ We assume $\Delta_{0}=\Delta_{0}^{s}$ in the case of $s$-wave pairing and $\Delta_{0}=\Delta_{0}^{d}$ in the case of $d$-wave pairing.

${ }^{27}$ A. Furusaki and M. Tsukada, Phys. Rev. B 43, 10164 (1991).

${ }^{28}$ R. Žikić, L. Dobrosavljević-Grujić and Z. Radović, Phys. Rev. B 59, 14644 (1999).

${ }^{29}$ A. Barone and G. Paterno, Physics and Applications of the Josephson Effect (John Wiley \& Sons, New York, 1982).

${ }^{30}$ S. V. Kuplevakhskĩ and I. I. Fal'ko, Zh. Eksp. Teor. Fiz., Pis'ma 52, 957 (1990) [JETP Lett., 52, 340 (1990)]. 
FIG. 1. Schematic illustration of the weak link. The fourfold symmetry of the gap functions is indicated.

FIG. 2. Reduced current $I / I_{0}$ as a function of phase difference $\phi$, for $\mathrm{S} / \mathrm{N} / \mathrm{S}, Z=0$ and $\mathrm{S} / \mathrm{F} / \mathrm{S}, Z=3$ weak links, at low temperature $t=0.05$, and at high temperature $t=5$. The reduced thickness is $\tilde{d}=0.25$. $2 \mathrm{D}$-wave pairing for three values of the misorientation angle $\theta$ (solid curves), and 3D s-wave pairing (dashed curves).

FIG. 3. Ground state phase difference across the link $\phi_{\mathrm{gs}}$ as a function of the ferromagnetic barrier influence $Z$, for $\theta=0$ and $\tilde{d}=0.25$. $2 \mathrm{D} d$-wave pairing (solid lines), and 3D $s$-wave pairing (dashed lines). These results are temperature independent.

FIG. 4. Ground state phase difference $\phi_{\mathrm{gs}}$ and reduced spontaneous current $I_{\mathrm{gs}} / I_{c}$ as functions of misorientation angle $\theta$ for a $d$-wave superconducting ring interrupted by $\mathrm{S} / \mathrm{N} / \mathrm{S}, Z=0$ and $\mathrm{S} / \mathrm{F} / \mathrm{S}, Z=3$ weak links, with $\tilde{d}=0.25$ and for three values of the reduced inverse inductance, $l^{-1}=0$ (open ring), 0.1 and 1 . Low temperature, $t=0.05$ (solid curves) and high temperature, $t=5$ (dashed curves).

FIG. 5. Highest spontaneous supercurrent $\left|I_{\mathrm{gs}}\right| / I_{c}$ as a function of $l^{-1}$ in a superconducting ring interrupted by a weak link. Reduced barrier thickness $\tilde{d}=0.25$. Low temperature, $t=0.05$ (solid curves): (1) $Z=0, \theta=\pi / 2$ and $(2) Z=3, \theta=0$ for $2 \mathrm{D} d$-wave pairing, and (3) $Z=3$ for $3 \mathrm{D} s$-wave pairing. High temperature, $t=5$ (dashed curve): universal behavior for all above cases .

FIG. 6. Magnetic flux $\Phi$ through a $d$-wave superconducting ring as a function of the misorientation angle $\theta$ for $t=0.05$, $\tilde{d}=0.25$, for two values of $l^{-1}$ and four values of the external magnetic flux $\Phi_{\mathrm{e}}$. S/N/S, $Z=0$, and S/F/S, $Z=3$ weak links. 
This figure "RadovicFig1.gif" is available in "gif" format from: http://arxiv.org/ps/cond-mat/9908092v1 
This figure "RadovicFig2.gif" is available in "gif" format from: http://arxiv.org/ps/cond-mat/9908092v1 
This figure "RadovicFig3.gif" is available in "gif" format from: http://arxiv.org/ps/cond-mat/9908092v1 
This figure "RadovicFig4.gif" is available in "gif" format from: http://arxiv.org/ps/cond-mat/9908092v1 
This figure "RadovicFig5.gif" is available in "gif" format from: http://arxiv.org/ps/cond-mat/9908092v1 
This figure "RadovicFig6.gif" is available in "gif" format from: http://arxiv.org/ps/cond-mat/9908092v1 